\title{
LEVEL RINGS ARISING FROM MEET-DISTRIBUTIVE MEET-SEMILATTICES
}

\author{
JÜRGEN HERZOG AND TAKAYUKI HIBI
}

\begin{abstract}
The homogenized ideal dual complex of an arbitrary meet-semilattice is introduced and described explicitly. Meet-distributive meet-semilattices whose homogenized ideal dual complex is level are characterized.
\end{abstract}

\section{Introduction}

In the present paper we continue our discussion in [6] and [5], and describe explicitly the generators of the homogenized ideal dual complex of an arbitrary meet-semilattice (Theorem 2.1). In case of meet-distributive meet-semilattices, a combinatorial formula (Proposition 1.2) to compute the $h$-vector of the homogenized ideal dual complex is given.

It is known [5] that the homogenized ideal dual complex $\Gamma_{\mathcal{L}}$ of a meetsemilattice $\mathcal{L}$ is Cohen-Macaulay if and only if $\mathcal{L}$ is meet-distributive. Thus it seems of interest to characterize the meet-distributive meet-semilattices $\mathcal{L}$ for which $\Gamma_{\mathcal{L}}$ is a level complex [8]. Our main theorem (Theorem 3.3) says that the homogenized ideal dual complex $\Gamma_{\mathcal{L}}$ is level if and only if a certain simplicial complex coming from $\mathcal{L}$ is pure. In particular, in case that $\mathcal{L}$ is a finite distributive lattice, $\Gamma_{\mathcal{L}}$ is level if and only if the simplicial complex consisting of all antichains of the poset of all join-irreducible elements of $\mathcal{L}$ is pure (Corollary 3.4).

\section{$\S 1$. The $h$-vector of a finite meet-distributive meet-semilattice}

First of all, we prepare notation and terminologies on finite lattices and finite posets (partially ordered sets). In a finite poset $P$ we say that $\alpha \in P$ covers $\beta \in P$ (or $\beta$ is a lower neighbor of $\alpha$ ) if $\beta<\alpha$ and $\beta<\gamma<\alpha$ for no $\gamma \in P$. Let $N(\alpha)$ denote the set of lower neighbors of $\alpha \in P$. A poset ideal

Received April 8, 2004.

Revised March 28, 2005, May 12, 2005.

1991 Mathematics Subject Classification: 13D02, 13H10, 06A12, 06D99. 
of $P$ is a subset $\mathcal{I}$ of $P$ such that $\alpha \in \mathcal{I}$ and $\beta \in P$ together with $\beta \leq \alpha$ imply $\beta \in \mathcal{I}$.

Let $\mathcal{L}$ be a finite meet-semilattice $[7$, p. 103] and $\hat{0}$ its unique minimal element. Since $\mathcal{L}$ is a meet-semilattice, it follows from [7, Proposition 3.3.1] that $\mathcal{L}$ is a lattice if and only if $\mathcal{L}$ possesses a unique maximal element $\hat{1}$. In other words, if $\mathcal{L}$ is a meet-semilattice and is not a lattice, then $\mathcal{L} \cup\{\hat{1}\}$ with a new element $\hat{1}$ such that $\alpha<\hat{1}$ for all $\alpha \in \mathcal{L}$ becomes a lattice. Thus, in a finite meet-semilattice $\mathcal{L}$, each element of $\mathcal{L}$ is the join of elements of $\mathcal{L}$. A join-irreducible element of $\mathcal{L}$ is an element $\alpha \in \mathcal{L}$ such that one cannot write $\alpha=\beta \vee \gamma$ with $\beta<\alpha$ and $\gamma<\alpha$. In other words, a join-irreducible element of $\mathcal{L}$ is an element $\alpha \in \mathcal{L}$ which covers exactly one element of $\mathcal{L}$.

Let $\mathcal{L}$ be a finite meet-semilattice and $P \subset \mathcal{L}$ the set of join-irreducible elements of $\mathcal{L}$. We will associate each element $\alpha \in \mathcal{L}$ with the subset

$$
\ell(\alpha)=\{p \in P: p \leq \alpha\}
$$

Thus $\ell(\alpha)$ is a poset ideal of $P$, and $\alpha \in \ell(\alpha)$ if and only if $\alpha$ is joinirreducible. Moreover, for $\alpha$ and $\beta$ belonging to $\mathcal{L}$, one has $\ell(\alpha)=\ell(\beta)$ if and only if $\alpha=\beta$.

Lemma 1.1. One has $\ell(\alpha \wedge \beta)=\ell(\alpha) \cap \ell(\beta)$ for all $\alpha, \beta \in \mathcal{L}$.

Proof. Let $\gamma=\alpha \wedge \beta$. Then $\ell(\gamma) \subset \ell(\alpha) \cap \ell(\beta)$. Since $\mathcal{L} \cup\{\hat{1}\}$ with a new element $\hat{1}$ is a lattice, if $\ell(\gamma) \neq \ell(\alpha) \cap \ell(\beta)$ and if $p \in(\ell(\alpha) \cap \ell(\beta)) \backslash \ell(\gamma)$, then $\delta=\gamma \vee p \in \mathcal{L}$ with $\gamma<\delta \leq \alpha$ and $\delta \leq \beta$. This contradicts $\gamma=\alpha \wedge \beta$.

Let $K$ be a field and $K[\mathbf{x}, \mathbf{y}]=K\left[\left\{x_{p}, y_{p}\right\}_{p \in P}\right]$ denote the polynomial ring in $2|P|$ variables over $K$ with each $\operatorname{deg} x_{p}=\operatorname{deg} y_{p}=1$. We associate each element $\alpha \in \mathcal{L}$ with the squarefree monomial

$$
u_{\alpha}=\left(\prod_{p \in \ell(\alpha)} x_{p}\right)\left(\prod_{p \in P \backslash \ell(\alpha)} y_{p}\right) \in K[\mathbf{x}, \mathbf{y}]
$$

and set

$$
H_{\mathcal{L}}=\left(u_{\alpha}\right)_{\alpha \in \mathcal{L}} \subset K[\mathbf{x}, \mathbf{y}]
$$

Since the ideal $H_{\mathcal{L}}$ is squarefree, there is a simplicial complex $\Sigma_{\mathcal{L}}$ on the vertex set $\left\{x_{p}, y_{p}\right\}_{p \in P}$ whose Stanley-Reisner ideal $I_{\Sigma_{\mathcal{L}}}$ coincides with $H_{\mathcal{L}}$. We call $\Sigma_{\mathcal{L}}$ the homogenized ideal complex of $\mathcal{L}$. 
Let $\Gamma_{\mathcal{L}}$ denote the Alexander dual ([2], [4]) of $\Sigma_{\mathcal{L}}$ and call $\Gamma_{\mathcal{L}}$ the homogenized ideal dual complex of $\mathcal{L}$. We write $\mathcal{F}\left(\Gamma_{\mathcal{L}}\right)$ for the set of facets (maximal faces) of $\Gamma_{\mathcal{L}}$. One has

$$
\mathcal{F}\left(\Gamma_{\mathcal{L}}\right)=\left\{F_{\alpha}: \alpha \in \mathcal{L}\right\}
$$

where

$$
F_{\alpha}=\left\{x_{q}: q \in P \backslash \ell(\alpha)\right\} \cup\left\{y_{q}: q \in \ell(\alpha)\right\}
$$

Hence,

$$
I_{\Gamma_{\mathcal{L}}}=\bigcap_{\alpha \in \mathcal{L}}\left(\left\{x_{p}: p \in \ell(\alpha)\right\} \cup\left\{y_{q}: q \in P \backslash \ell(\alpha)\right\}\right) .
$$

In particular $\Gamma_{\mathcal{L}}$ is a pure simplicial complex of dimension $|P|-1$.

A finite meet-semilattice $\mathcal{L}$ is called meet-distributive [7, p. 156] if each interval $[\alpha, \beta]=\{\gamma \in \mathcal{L}: \alpha \leq \gamma \leq \beta\}$ of $\mathcal{L}$ such that $\alpha$ is the meet of the lower neighbors of $\beta$ in $[\alpha, \beta]$ is boolean. For example, every poset ideal of a finite distributive lattice is a meet-distributive meet-semilattice.

Let $\mathcal{L}$ be an arbitrary finite meet-distributive meet-semilattice and, as before, $P \subset \mathcal{L}$ the set of join-irreducible elements of $\mathcal{L}$. The distributive closure of $\mathcal{L}$ is the finite distributive lattice $\mathcal{J}(P)$ consisting of all poset ideals of $P$ ordered by inclusion.

Recall that Birkhoff's fundamental structure theorem on finite distributive lattices [7, Theorem 3.4.1] guarantees that every finite distributive lattice is of the form $\mathcal{J}(P)$ for a unique finite poset $P$. In fact, if $P$ is the set of join-irreducible element of a finite distributive lattice $\mathcal{L}$, then $\mathcal{L}=\mathcal{J}(P)$.

It is not difficult to see that the map $\ell: \mathcal{L} \rightarrow \mathcal{J}(P)$ defined by $(1)$ is an embedding of meet-semilattices if and only if $\mathcal{L}$ is meet-distributive. Consult [3] for further information about meet-distributive lattices.

Proposition 1.2. Let $\mathcal{L}$ be a finite meet-distributive meet-semilattice and $\Gamma_{\mathcal{L}}$ its homogenized ideal dual complex. Let $h\left(\Gamma_{\mathcal{L}}\right)=\left(h_{0}, h_{1}, \ldots\right)$ be its $h$-vector. Then, for all $i$, one has

$$
h_{i}=|\{\alpha \in \mathcal{L}:|N(\alpha)|=i\}| .
$$

Proof. Let $\alpha \in \mathcal{L}$ with $|N(\alpha)|=i$ and $\ell(\alpha)=\left\{q_{1}, \ldots, q_{\delta}\right\}$. Let $N(\alpha)=$ $\left\{r_{1}, \ldots, r_{i}\right\}$ with each $\ell\left(r_{j}\right)=\ell(\alpha) \backslash\left\{q_{\delta-j+1}\right\}$. Let $r=r_{1} \wedge \cdots \wedge r_{i}$. Thus $\ell(r)=\bigcap_{j=1}^{i} \ell\left(r_{j}\right)=\left\{q_{1}, \ldots, q_{\delta-i}\right\}$ and the interval $[r, \alpha]$ in $\mathcal{L}$ is the boolean lattice of rank $i$. Since a subset $A \subset \ell(\alpha)$ is contained in none of the sets $\ell\left(r_{1}\right), \ldots, \ell\left(r_{i}\right)$ if and only if $A$ contains $\left\{q_{\delta}, q_{\delta-1}, \ldots, q_{\delta-i+1}\right\}$, it follows that 
the number of subsets $A \subset \ell(\alpha)$ with $|A|=k$ such that $A \subset \ell(q)$ for no $q \in \mathcal{L}$ with $q<\alpha$ is $\left(\begin{array}{c}\delta-i \\ k-i\end{array}\right)$. In other words, the number of those faces $F \subset F_{\alpha}$ of $\Gamma$ with $|F|=j+1$ such that $F \subset F_{q}$ for no $q \in \mathcal{L}$ with $q<\alpha$ is $\sum_{k=i}^{\delta}\left(\begin{array}{c}|P|-\delta \\ j-k+1\end{array}\right)\left(\begin{array}{c}\delta-i \\ k-i\end{array}\right)$, which is equal to $\left(\begin{array}{c}|P|-i \\ j-i+1\end{array}\right)=\left(\begin{array}{c}|P|-i \\ |P|-j-1\end{array}\right)$. Thus the number of faces $F$ of $\Gamma_{\mathcal{L}}$ with $|F|=j+1$ is

$$
f_{j}\left(\Gamma_{\mathcal{L}}\right)=\sum_{i=0}^{j+1}\left(\begin{array}{c}
|P|-i \\
|P|-j-1
\end{array}\right)|\{\alpha \in \mathcal{L}:|N(\alpha)|=i\}| .
$$

On the other hand, in general, one has

$$
f_{j}\left(\Gamma_{\mathcal{L}}\right)=\sum_{i=0}^{j+1}\left(\begin{array}{c}
|P|-i \\
|P|-j-1
\end{array}\right) h_{i}\left(\Gamma_{\mathcal{L}}\right) .
$$

Hence $h_{i}\left(\Gamma_{\mathcal{L}}\right)=|\{\alpha \in \mathcal{L}:|N(\alpha)|=i\}|$, as desired.

Corollary 1.3. Let $\mathcal{L}$ be a finite meet-distributive meet-semilattice, $P$ the set of join-irreducible elements of $\mathcal{L}$ and $\Gamma_{\mathcal{L}}$ the homogenized ideal dual complex of $\mathcal{L}$. Let $n=|P|$ and $\left(h_{0}, h_{1}, \ldots, h_{n}\right)$ the $h$-vector of $\Gamma_{\mathcal{L}}$. Then $h_{1}=n$, and the a-invariant of $\Gamma_{\mathcal{L}}$ (which is the nonpositive integer $\left.\max \left\{i: h_{i} \neq 0\right\}-n\right)$ is equal to $\max \{|N(\alpha)|: \alpha \in \mathcal{L}\}-n$.

ExAmple 1.4. Let $\mathcal{B}_{[n]}$ denote the boolean lattice of rank $n$ and $\mathcal{L}$ a poset ideal of $\mathcal{B}_{[n]}$ which contains all join-irreducible elements (i.e., $\{1\}, \ldots$, $\{n\})$ of $\mathcal{B}_{[n]}$. Then the meet-distributive meet-semilattice $\mathcal{L}$ is a simplicial complex on $[n]$ and the $h$-vector of $\Gamma_{\mathcal{L}}$ coincides with the $f$-vector of $\mathcal{L}$.

(a) By using (2) the Stanley-Reisner ideal $I_{\Gamma_{\mathcal{L}}}$ of $\Gamma_{\mathcal{L}}$ is generated by those squarefree monomials $\prod_{q \in \ell(\beta)} y_{q}$ such that $\beta \in \mathcal{B}_{[n]}$ is a minimal nonface of $\mathcal{L}$ and by the quadratic monomials $x_{\{i\}} y_{\{i\}}$ for all $i \in[n]$.

(b) Let $T=K\left[y_{\{1\}}, \ldots, y_{\{n\}}\right]$ and $J \subset T$ the ideal generated by those squarefree monomials $\prod_{q \in \ell(\beta)} y_{q}$ such that $\beta \in \mathcal{B}_{[n]}$ is a minimal nonface of $\mathcal{L}$ and by $y_{\{i\}}^{2}$ for all $i \in[n]$. The quotient ring $T / J$ is 0 -dimensional and its $h$-vector is $\left(f_{-1}, f_{0}, \ldots\right)$, the $f$-vector of $\mathcal{L}$ with $f_{-1}=1$. It turns out that $I_{\Gamma_{\mathcal{L}}}$ is the polarization [1, Lemma 4.3.2] of the ideal $J$. Since $T / J$ is Cohen-Macaulay, it follows immediately that $\Gamma_{\mathcal{L}}$ is Cohen-Macaulay. This fact is a special case of [5, Corollary 1.6].

(c) Since $T / J$ is a level ring $[8$, p. 91] if and only if the simplicial complex $\mathcal{L}$ is pure, it follows that the homogenized ideal dual complex $\Gamma_{\mathcal{L}}$ of $\mathcal{L}$ is a level complex if and only if the simplicial complex $\mathcal{L}$ is pure. 
(d) Let $\Delta$ be a simplicial complex on the vertex set $V=\left\{y_{\{1\}}, \ldots, y_{\{n\}}\right\}$, and let $W=\left\{x_{\{1\}}, \ldots, x_{\{n\}}\right\}$. We write $\Delta^{\sharp}$ for the simplicial complex on the vertex set $V \cup W$ whose facets are those of $\Delta$ together with all edges $\left\{x_{\{i\}}, y_{\{i\}}\right\}$ for $i=1, \ldots, n$. By the observation (a) for a simplicial complex $\mathcal{L}\left(\subset \mathcal{B}_{[n]}\right)$ on $[n]$ one has a simplicial complex $\Delta$ on $V$ such that the facet ideal of $\Delta^{\sharp}$, i.e., the ideal generated by all monomials corresponding to the facets, coincides with the Stanley-Reisner ideal $I_{\Gamma_{\mathcal{L}}}$ of $\Gamma_{\mathcal{L}}$. Conversely, given a simplicial complex $\Delta$ on $V$, there is a simplicial complex $\mathcal{L}\left(\subset \mathcal{B}_{[n]}\right)$ on $[n]$ such that the facet ideal of $\Delta^{\sharp}$ coincides with $I_{\Gamma_{\mathcal{L}}}$. Since $\Gamma_{\mathcal{L}}$ is always Cohen-Macaulay, the facet ideal of $\Delta^{\sharp}$ is Cohen-Macaulay. This argument is a direct and easy proof of [5, Corollary 4.4].

(e) By using (b) and (c), it follows that every $f$-vector of a pure simplicial complex is the $h$-vector of a level complex.

It would, of course, be of interest to generalize the fact (c) of Example 1.4 to arbitrary meet-distributive meet-semilattices $\mathcal{L}$.

\section{$\S 2$. Alexander duality of meet-distributive meet-semilattices}

A nice description of the homogenized ideal dual complex of a finite distributive lattice is obtained in [6, Lemma 3.1]. On the other hand, the homogenized ideal dual complex of a meet-distributive meet-semilattice of a special kind, namely, a poset ideal of a finite distributive lattice is described in [5, Theorem 4.2]. An explicit description of the homogenized ideal dual complex of an arbitrary finite meet-semilattice will be obtained in Theorem 2.1 below.

If, in general, $P$ is a finite poset and $B \subset P$, then we write $\langle B\rangle$ for the poset ideal of $P$ generated by $B$, i.e., $p \in P$ belongs to $\langle B\rangle$ if and only if $p \leq q$ for some $q \in B$.

THEOREM 2.1. Let $\mathcal{L}$ be an arbitrary finite meet-semilattice and $P$ the set of join-irreducible elements of $\mathcal{L}$. Then the Stanley-Reisner ideal $I_{\Gamma_{\mathcal{L}}}$ of the homogenized ideal dual complex $\Gamma_{\mathcal{L}}$ of $\mathcal{L}$ is generated by the following squarefree monomials:

(i) $x_{p} y_{q}$, where $p, q \in P$ with $p<q$;

(ii) $\prod_{q \in B} y_{q}$, where $B$ is an antichain of $P$ with $\langle B\rangle \not \subset \ell(\alpha)$ for all $\alpha \in \mathcal{L}$;

(iii) $x_{p} \prod_{q \in B} y_{q}$, where $B$ is an antichain of $P$ with $\ell(\beta) \neq\langle B\rangle$ for all $\beta \in$ $\mathcal{L}$, but with $\langle B\rangle \subset \ell(\alpha)$ for some $\alpha \in \mathcal{L}$ and where $p \in \ell\left(\bigwedge_{\langle B\rangle \subset \ell(\alpha)} \alpha\right) \backslash$ $\langle B\rangle$. 
Proof. Let $A \subset P$ and $B \subset P$ with $A \cap B=\emptyset$. We write $\mathbf{x}_{A} \mathbf{y}_{B}$ for the squarefree monomial $\prod_{p \in A} x_{p} \prod_{q \in B} y_{q}$ of $K[\mathbf{x}, \mathbf{y}]$. By the definition of the Stanley-Reisner ideal $I_{\Gamma_{\mathcal{L}}}$ of $\Gamma_{\mathcal{L}}$, it follows that $\mathbf{x}_{A} \mathbf{y}_{B}$ belongs to $I_{\Gamma_{\mathcal{L}}}$ if and only if there is no facet $\mathcal{F}_{\alpha}$ of $\Gamma_{\mathcal{L}}$ with $\left\{x_{p}: p \in A\right\} \cup\left\{y_{q}: q \in B\right\} \subset \mathcal{F}_{\alpha}$. Thus by using (2) one has $\mathbf{x}_{A} \mathbf{y}_{B} \in I_{\mathcal{L}_{\Gamma}}$ if and only if there is no $\alpha \in \mathcal{L}$ such that $A \subset P \backslash \ell(\alpha)$ and $B \subset \ell(\alpha)$. In other words, one has $\mathbf{x}_{A} \mathbf{y}_{B} \in I_{\mathcal{L}_{\Gamma}}$ if and only if the following condition $(*)$ is satisfied:

$$
\text { each } \alpha \in \mathcal{L} \text { with } B \subset \ell(\alpha) \text { satisfies } A \cap \ell(\alpha) \neq \emptyset \text {. }
$$

We say that a pair $(A, B)$, where $A \subset P$ and $B \subset P$ with $A \cap B=\emptyset$, is an independent pair of $\mathcal{L}$ if the condition $(*)$ is satisfied. Thus $I_{\mathcal{L}_{\Gamma}}$ is generated by all monomials $x_{p} y_{p}$ with $p \in P$ together with those monomials $\mathbf{x}_{A} \mathbf{y}_{B}$ such that $(A, B)$ is an independent pair of $\mathcal{L}$.

Let $M(B)$ denote the set of maximal elements of $B$. Thus one has $\langle B\rangle=\langle M(B)\rangle$. Hence $(A, B)$ is independent if and only if $(A, M(B))$ is independent. Since $\mathbf{x}_{A} \mathbf{y}_{M(B)}$ divides $\mathbf{x}_{A} \mathbf{y}_{B}$ and since $M(B)$ is an antichain of $P$, it follows that $I_{\mathcal{L}_{\Gamma}}$ is generated by all monomials $x_{p} y_{p}$ with $p \in P$ together with those monomials $\mathbf{x}_{A} \mathbf{y}_{B}$ such that $(A, B)$ is an independent pair of $\mathcal{L}$ and $B$ is an antichain of $P$.

Let $p$ and $q$ belong to $P$. Since $\ell(q)=\langle\{q\}\rangle \in \mathcal{L}$ for all $q \in \mathcal{L}$, the pair $(\{p\},\{q\})$ with $p \neq q$ is an independent pair of $\mathcal{L}$ if and only if $p<q$. Let $\ell(\beta)=\langle B\rangle$ for some $\beta \in \mathcal{L}$. Then a pair $(A, B)$ is independent if and only if $A \cap\langle B\rangle \neq \emptyset$. On the other hand, $A \cap\langle B\rangle \neq \emptyset$ if and only if there are $p \in A$ and $q \in B$ with $p<q$.

Consequently, $I_{\mathcal{L}_{\Gamma}}$ is generated by all monomials $x_{p} y_{q}$, where $p, q \in$ $P$ with $p<q$ together with those monomials $\mathbf{x}_{A} \mathbf{y}_{B}$, where $(A, B)$ is an independent pair of $\mathcal{L}$ such that $B$ is an antichain of $P$ with $\ell(\beta)=\langle B\rangle$ for no $\beta \in \mathcal{L}$ and with $A \cap\langle B\rangle=\emptyset$.

Now, let $B$ be an antichain of $P$ with $\ell(\beta)=\langle B\rangle$ for no $\beta \in \mathcal{L}$ and $A \subset P$ with $A \cap\langle B\rangle=\emptyset$.

(a) First, if $\langle B\rangle \subset \ell(\alpha)$ for no $\alpha \in \mathcal{L}$, then $(A, B)$ is independent for all $A \subset P$ with $A \cap B=\emptyset$. Thus in particular $(\emptyset, B)$ is an independent pair of $\mathcal{L}$.

(b) Second, if $\langle B\rangle \subset \ell(\alpha)$ for some $\alpha \in \mathcal{L}$, then $(A, B)$ is independent if and only if $A \cap\left(\bigcap_{\langle B\rangle \subset \ell(\alpha)} \ell(\alpha)\right) \neq \emptyset$. Since $\bigcap_{\langle B\rangle \subset \alpha} \ell(\alpha)=\ell\left(\bigwedge_{\langle B\rangle \subset \alpha} \alpha\right)$, it follows that $(A, B)$ is independent if and only if there is $p \in A$ with $p \in \ell\left(\bigwedge_{\langle B\rangle \subset \alpha} \alpha\right) \backslash\langle B\rangle$. 


\section{§3. Level rings arising from meet-distributive meet-semilattices}

It is known [5, Corollary 1.6] that the homogenized ideal dual complex $\Gamma_{\mathcal{L}}$ of a finite meet-semilattice $\mathcal{L}$ is Cohen-Macaulay if and only if $\mathcal{L}$ is meetdistributive. The problem when the homogenized ideal dual complex of a meet-distributive meet-semilattice is a level ring is now studied.

Recall that a Cohen-Macaulay graded ring $R=R_{0} \oplus R_{1} \oplus \cdots$ over a field $K=R_{0}$ is called level [8, p. 91] if the canonical module of $R$ is generated in one degree. Every Gorenstein ring is level.

Let $\mathcal{L}$ be a finite meet-distributive meet-semilattice and $P$ the set of join-irreducible elements of $\mathcal{L}$. Let, as before, $K[\mathbf{x}, \mathbf{y}]=K\left[\left\{x_{p}, y_{p}\right\}_{p \in P}\right]$ denote the polynomial ring in $2|P|$ variables over a field $K$ with each $\operatorname{deg} x_{p}=\operatorname{deg} y_{p}=1$.

For each $\alpha \in \mathcal{L}$ we write $\alpha^{\prime} \in \mathcal{L}$ for the meet of all $\beta \in N(\alpha)$, where $N(\alpha)$ is the set of lower neighbors of $\alpha$. Since $\mathcal{L}$ is a meet-distributive meet-semilattice, it follows that the interval

$$
\mathcal{B}_{\alpha}=\left[\alpha^{\prime}, \alpha\right]=\left\{\gamma \in \mathcal{L}: \alpha^{\prime} \leq \gamma \leq \alpha\right\}
$$

of $\mathcal{L}$ is a boolean lattice. Let $S(\alpha) \subset P$ denote the antichain

$$
S(\alpha)=\ell(\alpha) \backslash \ell\left(\alpha^{\prime}\right) \text {. }
$$

Each element belonging to $S(\alpha)$ is a maximal element of $\ell(\alpha)$, and $t \in \ell(\alpha)$ belongs to $S(\alpha)$ if and only if $\ell(\beta)=\ell(\alpha) \backslash\{t\}$ for some $\beta \in N(\alpha)$.

Lemma 3.1. If $\alpha$ and $\beta$ belong to $\mathcal{L}$ with $\alpha \neq \beta$, then $S(\alpha) \neq S(\beta)$.

Proof. Let $\gamma=\alpha \wedge \beta$. If $S(\alpha)=S(\beta)$, then $S(\alpha) \subset S(\gamma)$. In fact, for each $t \in S(\alpha)=S(\beta)$, there are $\alpha_{0} \in N(\alpha)$ and $\beta_{0} \in N(\beta)$ with $\ell\left(\alpha_{0}\right)=\ell(\alpha) \backslash\{t\}$ and $\ell\left(\beta_{0}\right)=\ell(\beta) \backslash\{t\}$. By using Lemma 1.1, one has $\ell\left(\alpha_{0} \wedge \beta_{0}\right)=\ell(\gamma) \backslash\{t\}$. Hence $t \in S(\gamma)$. Now, since $\gamma<\alpha$, one has $\delta \in N(\alpha)$ with $\gamma \leq \delta<\alpha$. Since $\ell(\delta)=\ell(\alpha) \backslash\{t\}$ for some $t \in S(\alpha)$, it follows that $t \notin \ell(\gamma)$. This contradict $S(\alpha) \subset S(\gamma)$. Hence $S(\alpha) \neq S(\beta)$, as desired.

Recall from the proof of Theorem 2.1 that a pair $(A, B)$, where $A \subset P$ and $B \subset P$ with $A \cap B=\emptyset$, is said to be an independent pair of $\mathcal{L}$ if each $\alpha \in \mathcal{L}$ with $B \subset \ell(\alpha)$ satisfies $A \cap \ell(\alpha) \neq \emptyset$.

Lemma 3.2. Let $\alpha \in \mathcal{L}$ and $T \subset S(\alpha)$. Then the pair $(\emptyset, T)$ cannot be independent. Moreover, for $p \in S(\alpha) \backslash T$, the pair $(\{p\}, T)$ cannot be independent. 
Proof. Since $T \subset \ell(\alpha)$, the pair $(\emptyset, T)$ cannot be an independent pair of $\mathcal{L}$. On the other hand, since $p \in S(\alpha)$, one has $\beta \in N(\alpha)$ with $\ell(\beta)=$ $\ell(\alpha) \backslash\{p\}$. Since $T \subset \ell(\beta)$ and since $\{p\} \cap \ell(\beta)=\emptyset$, it follows that $(\{p\}, T)$ cannot be an independent pair of $\mathcal{L}$, as desired.

Let $I_{\Gamma_{\mathcal{L}}}$ denote the Stanley-Reisner ideal of $\mathcal{L}$ and $K\left[\Gamma_{\mathcal{L}}\right]=K[\mathbf{x}, \mathbf{y}] / I_{\Gamma_{\mathcal{L}}}$ the Stanley-Reisner ring of $\Gamma_{\mathcal{L}}$. Since the dimension of $\Gamma_{\mathcal{L}}$ is $|P|-1$ and the Krull dimension of $K\left[\Gamma_{\mathcal{L}}\right]$ coincides with $|P|$, it follows easily that $\left\{x_{p}-y_{p}\right.$ : $p \in P\}$ is a linear system of parameters of $K\left[\Gamma_{\mathcal{L}}\right]$. Since $K\left[\Gamma_{\mathcal{L}}\right]$ is CohenMacaulay, by using Proposition 1.2, the Hilbert series of the quotient ring

$$
K\left[\Gamma_{\mathcal{L}}\right] /\left(x_{p}-y_{p}: p \in P\right)
$$

is $h_{0}+h_{1} \lambda+h_{2} \lambda^{2}+\cdots$, where $\left(h_{0}, h_{1}, h_{2}, \ldots\right)$ is the $h$-vector of $\Gamma_{\mathcal{L}}$.

Let $J_{\Gamma_{\mathcal{L}}}$ be the monomial ideal of $K[\mathbf{x}]=K\left[\left\{x_{p}\right\}_{p \in P}\right]$ generated by those monomials

(i) $x_{p} x_{q}$, where $p, q \in P$ with $p<q$;

(ii) $\prod_{q \in B} x_{q}$, where $B$ is an antichain of $P$ with $\langle B\rangle \subset \ell(\alpha)$ for no $\alpha \in \mathcal{L}$;

(iii) $x_{p} \prod_{q \in B} x_{q}$, where $B$ is an antichain of $P$ with $\ell(\beta)=\langle B\rangle$ for no $\beta \in$ $\mathcal{L}$, but with $\langle B\rangle \subset \ell(\alpha)$ for some $\alpha \in \mathcal{L}$ and where $p \in \ell\left(\bigwedge_{\langle B\rangle \subset \ell(\alpha)} \alpha\right) \backslash$ $\langle B\rangle$.

By virtue of Theorem 2.1 it follows that

$$
K[\mathbf{x}] / J_{\Gamma_{\mathcal{L}}}=K\left[\Gamma_{\mathcal{L}}\right] /\left(x_{p}-y_{p}: p \in P\right)
$$

We associate each $\alpha \in \mathcal{L}$ with the monomial

$$
u_{\alpha}=\prod_{p \in S(\alpha)} x_{p}
$$

of degree $|N(\alpha)|$.

TheOREM 3.3. Let $\mathcal{L}$ be a finite meet-distributive meet-semilattice, $P$ the set of join-irreducible elements of $\mathcal{L}$, and $K[\mathbf{x}]=K\left[\left\{x_{p}\right\}_{p \in P}\right]$ the polynomial ring in $|P|$ variables over a field $K$. Then the set of monomials $\left\{u_{\alpha} ; \alpha \in \mathcal{L}\right\}$ is a $K$-basis of the quotient ring $K[\mathbf{x}] / J_{\Gamma_{\mathcal{L}}}$. Thus in particular $\{S(\alpha): \alpha \in \mathcal{L}\}$ is a simplicial complex on the vertex set $\left\{x_{p}: p \in P\right\}$ whose $f$-vector coincides with the $h$-vector of $\mathcal{L}$. 
Proof. Lemma 3.2 says that, for each $\alpha \in \mathcal{L}$, the monomial $u_{\alpha}$ does not belongs to $J_{\Gamma_{\mathcal{L}}}$. Moreover, Lemma 3.1 guarantees that, for $\alpha \neq \beta$ belonging to $\mathcal{L}$, one has $u_{\alpha} \neq u_{\beta}$. Hence, for each $i=0,1,2, \ldots$, the number of monomials $u_{\alpha}$ with $\alpha \in \mathcal{L}$ of degree $i$ is equal to $h_{i}$. Since the Hilbert series of $K[\mathbf{x}] / J_{\Gamma_{\mathcal{L}}}$ is $h_{0}+h_{1} \lambda+h_{2} \lambda^{2}+\cdots$, it follows that $\left\{u_{\alpha} ; \alpha \in \mathcal{L}\right\}$ is a $K$-basis of $K[\mathbf{x}] / J_{\Gamma_{\mathcal{L}}}$, as required.

We now come to the combinatorial characterization for the homogenized ideal dual complex $\Gamma_{\mathcal{L}}$ of a finite meet-distributive meet-semilattice $\mathcal{L}$ to be level.

Corollary 3.4. The homogenized ideal dual complex $\Gamma_{\mathcal{L}}$ of a finite meet-distributive meet-semilattice $\mathcal{L}$ is a level complex if and only if the simplicial complex $\{S(\alpha): \alpha \in \mathcal{L}\}$ is pure. Thus in particular the homogenized ideal dual complex $\Gamma_{\mathcal{L}}$ of a finite distributive lattice $\mathcal{L}=\mathcal{J}(P)$ is level if and only if the simplicial complex consisting of all antichains of $P$ is pure.

Consider the following example of a meet-distributive meet-semilattice $\mathcal{L}$

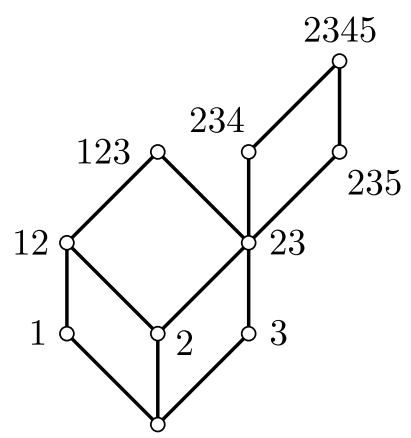

with the following poset of join-irreducible elements

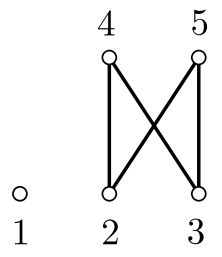

By using Theorem 2.1 the Stanley-Reisner ideal of the homogenized ideal dual complex of $\mathcal{L}$ is generated by the following monomials: 
(i) $x_{1} y_{1}, \ldots, x_{5} y_{5}, x_{2} y_{4}, x_{2} y_{5}, x_{3} y_{4}$ and $x_{3} y_{5}$;

(ii) $y_{1} y_{4}$ and $y_{1} y_{5}$;

(iii) $x_{2} y_{1} y_{3}$.

The $h$-vector of $\mathcal{L}$ is $(1,5,4)$. By using Corollary 3.4 the homogenized ideal dual complex $\Gamma_{\mathcal{L}}$ is level.

In the following meet-distributive meet-semilattice $\mathcal{L}$ the facets of the simplicial complex $\{S(\alpha): \alpha \in \mathcal{L}\}$ are $\{1,2\},\{1,3,4\}$ and $\{2,3,4\}$. Since this simplicial complex is not pure it follows from Corollary 3.4 that $\Gamma_{\mathcal{L}}$ is not level. The $h$-vector of $\mathcal{L}$ is $(1,4,6,2)$.

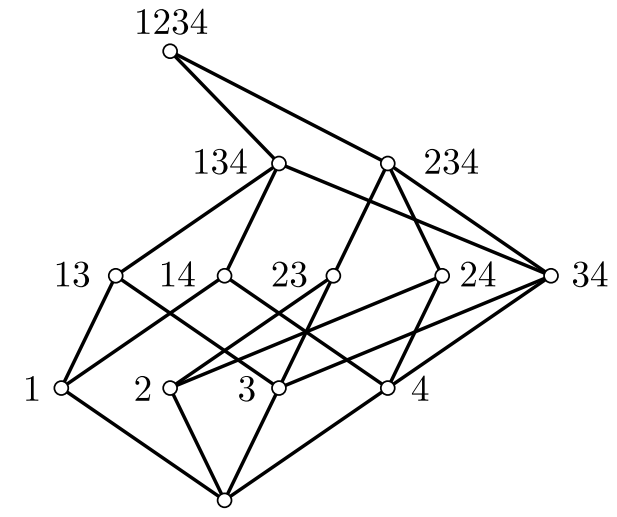

By Theorem 3.3 the $h$-vector of the homogenized ideal dual complex of a meet-distributive meet-semilattice is just the $f$-vector of a simplicial complex, and the $h$-vector of a level simplicial complex coming from a meetdistributive meet-semilattice is just the $f$-vector of a pure simplicial complex. These facts lead us to the following

Question 3.5. (a) Characterize the $h$-vectors of the homogenized ideal dual complex of finite distributive lattices.

(b) Characterize the $h$-vectors of the homogenized ideal dual complex of meet-distributive lattices.

(c) Find a nice class of level simplicial complexes whose $h$-vector is not the $f$-vector of a pure simplicial complex.

For example $(1,3,3)$ is the $h$-vector of the the homogenized ideal dual complex of the meet-distributive lattice $\mathcal{B}_{[3]} \backslash\{1,3\}$, but is not the $h$-vector of the homogenized ideal dual complex of a distributive lattice. 


\section{REFERENCES}

[1] W. Bruns and J. Herzog, Cohen-Macaulay rings, Revised Edition, Cambridge University Press, 1996.

[2] J. Eagon and V. Reiner, Resolutions of Stanley-Reisner rings and Alexander duality, J. Pure Appl. Algebra, 130 (1998), 265-275.

[3] P. Edelman, Abstract convexity and meet-distributive lattices, Combinatorics and ordered sets (Arcata, Calif., 1985), Contemp. Math. 57, Amer. Math. Soc., Providence, RI (1986), pp. 127-150.

[4] J. Herzog, T. Hibi and X. Zheng, Dirac's theorem on chordal graphs and Alexander duality, Europ. J. Combin., 25 (2004), 949-960.

[5] J. Herzog, T. Hibi and X. Zheng, The monomial ideal of a finite meet-semilattice, to appear, Trans. Amer. Math. Soc.

[6] J. Herzog and T. Hibi, Distributive Lattices, Bipartite Graphs and Alexander Duality, to appear, J. Alg. Combin.

[7] R. P. Stanley, Enumerative Combinatorics, Volume I, Wadsworth \& Brooks/Cole, Monterey, CA, 1986.

[8] R. P. Stanley, Combinatorics and Commutative Algebra, Second Edition, Birkhäuser, Boston, MA, 1996.

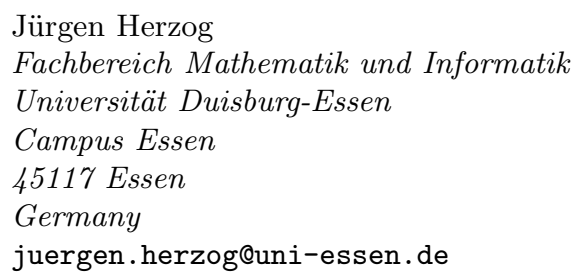

Jürgen Herzog

Fachbereich Mathematik und Informatik

Universität Duisburg-Essen

Campus Essen

45117 Essen

Germany

juergen.herzog@uni-essen.de

Takayuki Hibi

Department of Pure and Applied Mathematics

Graduate School of Information Science and Technology

Osaka University

Toyonaka

Osaka 560-0043

Japan

hibi@math.sci.osaka-u.ac.jp 